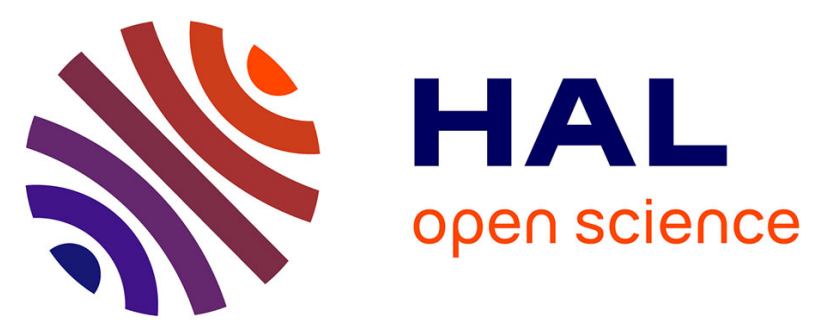

\title{
Color texture classification method based on a statistical multi-model and geodesic distance
}

\author{
Ahmed Drissi El Maliani, Mohammed El Hassouni, Yannick Berthoumieu, \\ Driss Aboutajdine
}

\section{- To cite this version:}

Ahmed Drissi El Maliani, Mohammed El Hassouni, Yannick Berthoumieu, Driss Aboutajdine. Color texture classification method based on a statistical multi-model and geodesic distance. Journal of Visual Communication and Image Representation, 2014, 25 (7), pp.1717-1725. 10.1016/j.jvcir.2014.06.004 . hal-01015628

\section{HAL Id: hal-01015628 \\ https://hal.science/hal-01015628}

Submitted on 31 Jan 2018

HAL is a multi-disciplinary open access archive for the deposit and dissemination of scientific research documents, whether they are published or not. The documents may come from teaching and research institutions in France or abroad, or from public or private research centers.
L'archive ouverte pluridisciplinaire HAL, est destinée au dépôt et à la diffusion de documents scientifiques de niveau recherche, publiés ou non, émanant des établissements d'enseignement et de recherche français ou étrangers, des laboratoires publics ou privés. 


\title{
Color texture classification method based on a statistical multi-model and geodesic distance
}

\author{
Ahmed Drissi El Maliani ${ }^{\mathrm{a}}$, Mohammed El Hassouni ${ }^{\mathrm{b}, \mathrm{d},}$, Yannick Berthoumieu ${ }^{\mathrm{c}}$, Driss Aboutajdine ${ }^{\mathrm{d}}$, \\ ${ }^{a}$ L.I.M. Faculty of Sciences Dhar el Mahraz, USMBA, Fs, Morocco \\ ${ }^{b}$ DESTEC, FLSHR, University of Mohammed V-Agdal, Rabat, Morocco. \\ ${ }^{c}$ Institut Polytechnique Bordeaux/ENSEIRB-MATMECA, Laboratoire IMS CNRS UMR 5218 - Groupe Signal et Image, France. \\ ${ }^{d}$ LRIT URAC 29, University of Mohammed V-Agdal, Rabat, Morocco.
}

\begin{abstract}
In this paper, we propose a novel color texture classification method based on statistical characterization. The approach consists in modeling complex wavelet coefficients of both luminance and chrominance components separately leading to a multi-modeling approach. The copula theory allows to take into account the spatial dependencies which exist within the intra-luminance sub-bands via the luminance model $M_{L}$, and also between the inter-chrominance subband coefficients via the chrominance model $M_{C_{r}}$. The multi-model, i.e $M_{L}$ and $M_{C_{r}}$, is used to develop a Bayesian classifier based on the softmax principal. To derive the classifier, we propose a closed-form expression for the Rao geodesic distance between two copulas. Experiments on two sub-families of luminance-chrominance color spaces namely Lab and HSV have been carried out for a wide range of color texture databases. The combination of different statistical sub-models show that the multi-modeling performs better than some existing methods in term of classification rates.
\end{abstract}

Keywords: Color texture, Wavelet representation, Multivariate copula model, Rao geodesic distance, Bayesian classification

\section{Introduction}

For color texture analysis, the question of the color space selection to characterize the visual content may be raised. RGB space is the most natural since it represents the acquisition-reference space. However, R, G and B channels are known to exhibit simultaneously high statistical dependencies relatively to: 1) the intra-channel or spatial one, 2) the inter-band for scale-space representation one and 3) the inter-channel or multicomponent one. In recent works, some authors have proposed various stochastic models to characterize the inter-channel dependencies alone. From scale-space representation such as 2-D wavelet or others, previous approaches consist in fitting the histograms of the wavelet subbands with a given parametric probability density function (pdf) of the trivariate color vector samples for each level of the decomposition. Most important recent works for RGB color space are based on statistical approach based on multivariate parametric tests (Seetharama et al. 2014), multivariate generalized Gaussian distribution in (Verdoolaege et al. 2012) and copulas based multivariate Weibull distribution (Kwitt et al. 2011). The parameters of the pdf are the covariance matrix and different auxiliary parameters such as the shape parameter characterizing the non-Gaussiannity.

Email addresses: maliani.ahmed@gmail.com (Ahmed Drissi El Maliani), mohamed.elhassouni@gmail.com (Mohammed El Hassouni)
Extending these models to take into account jointly intraband, inter-band and inter-channel dependencies, leads to a high-dimensional model in terms of number of parameters. This is a main drawback of these proposals since, in practice, for a fixed data size, dealing with high-dimensional model may be a critical issue in terms of parameter estimation performance. Considering color space, another alternative to the RGB space is to consider the family of luminance-chrominance (LC) color spaces (DeYoe et al. 1996; Mojsilovic et al. 2000). This family includes perceptual (HSV, HSI and HSB) and perceptually uniform (Lab, Yuv, YCbCr) color spaces (Sarifuddin et al. 2005). An extended comparison of those spaces is given by (Qazi et al. 2011). These authors note that the luminance channel is uncorrelated with the chrominance channels. From this independence in LC spaces, they proposed a model taking into account separately intra and inter dependencies, i.e. multi-model, with few parameters compared to the required number by using the RGB space. Qazi et al. proposed to consider a multimodel based on a 2-D complex quarter plan autoregressive (2-D QP AR) model for luminance as well as for chrominance information. This may lead to a lack of flexibility because the luminance and chrominance are uncorrelated and thus, have distributions of different natures.

In this paper, considering the LC color spaces, we propose a multi-modeling approach based on multivariate stochastic modeling. The contribution is twofold: 
- First, we propose a multi-model based on two nonGaussian sub-models, i.e. $M_{L}$ and $M_{C_{r}}$, devoted to describe respectively 1 ) the intra-channel dependency by considering the spatial structure and interband dependence of the luminance channel, and 2) the inter-channel dependency by considering a bivariate pdf for the two chrominance channels. Both $M_{L}$ and $M_{C_{r}}$ are based on copula as a powerful tool to construct non-Gaussian joint models that fit data with even different natures of margins. Linear and/or circular models are derived depending on the component natures (ex: the Hue in HSV color space is a circular channel).

- Second, for the similarity measurement used to compare two texture samples, we derive a closed form expression for the Rao geodesic distance for copula based multivariate models. Contrary to the conventional Kullback-leibler (KL) divergence, the Rao geodesic metric has the advantage to satisfy distance properties such as symmetry and triangular inequality (Atkinson et al. 1981).

The use of different models for characterizing the wavelet coefficients has been recently investigated by (Rakvongthai et al. 2013), for texture retrieval in a noisy environment. Authors proposed different univariate statistical models for the real/imaginary, magnitude and phase parts of complex subband coefficients. The parameter vector of the studied texture is then formed by the concatenation of the estimated parameters from each of these models. Also, the similarity measure has been expressed by a formula combining both magnitude and phase KL divergences using a mixing parameter. While the cited work did not consider the color information at all, it should be emphasized that the present work considers the color and texture information jointly for image characterization. Concerning the similarity measure, the originality of our method is the use of Rao geodesic distance which represents a good alternative to the KL divergence since it satisfies the distance properties. For this, a closed-form expression of Rao geodesic distance between copula based distributions is developed.

Note also that this work stands out from our previous work (Maliani et al. 2012) which presents a preliminary study of the multi-modeling concept that considered only the special case of color representation, the HSV color space. Moreover, the paper did not discuss the dimensionality reduction issue when considering the three dependencies (intra-band, inter-band and inter-channel). Also, in the classification step, we proposed the K-Nearest Neighborhood classifier taking the weighted sum of the two similarity measures between the luminance and chrominance sub-models. The weights have been tuned experimentally which is the same as for (Rakvongthai et al. 2013), which led us to test exhaustively on several scales of weights to get the best classification rate. The multi-modeling proposed in this paper, helps in dimensionality reduction of the extracted features to characterize the textural content. According to several dependence experiments presented in section 2, the spatial structure of the texture and the inter-band dependence is only considered for the luminance component and the inter color-channel dependency consists of a bivariate model. The performance of the multi-model is tested using a Bayesian classifier based on the softmax principal (Bishop 2006) thanks to its suitability to combine several similarity measures through a posteriori probability fusion. The experimental protocol adopted here, aims to find the optimal configuration of our multi-model. This starts with the choice of statistical distribution related to color components in the field of dual-tree complex wavelet CT-DWT (Kingsbury 1998). We proposed to build joint models by testing different combinations of Gamma, Weibull and VonMises distributions for both luminance and chrominance components. First comparisons were considered to show the relevance of each model for the color spaces. Then, we compared our best selected models with the state of the art methods in terms of classification rate, the dimensionality reduction and the computation time.

This paper is organized as follows. Section 2 deals with the proposed multi-modeling approach for textures in LC color spaces. In Section 3, we present the classification framework, and the proposed similarity measurement. Then, we show experimental results in Section 4. Finally, we give concluding remarks in Section 5 .

\section{Color texture modeling in LC color spaces}

\subsection{Dependence characterization}

Let us consider a stationary color texture $x_{T}$ represented in a given $\mathrm{LC}$ color space, and let $l$ be the luminance component, $c r_{1} / c r_{2}$ respectively the first and the second chrominance component of $x_{T}$. Each color component is first decomposed into a set of wavelet subbands. In wavelet domain texture characterization, it is common to model the statistical distribution of wavelet coefficients in different subbands, which corresponds to the detail subbands. The low-pass wavelet coefficients are excluded ((Mallat 1999), (Boubchir et al. 2010)). For 2-D wavelet transforms, each scale usually has several subbands corresponding to the different orientations. Let us consider $K$ subbands, where $K=S \times O$ with $S$ the number of scales and $O$ the number of orientations. The subbands are respectively $l^{(k)}, c r_{1}^{(k)}, c r_{2}^{(k)}$, $k=1, \cdots, K$. The statistical distribution of wavelet detail coefficients of natural textures has been extensively studied ((Simoncelli 1997), (Boubchir et al. 2010), (Lasmar et al. 2010), (Verdoolaege et al. 2012), (Kwitt et al. 2011)). It has been shown that the empirical distribution is generally heavy-tailed with a sharp peak at zero. This leptokurtic behavior leads to consider non-Gaussian marginal or joint density. Contrary to the marginal approach, the joint modeling needs to 
define the dependence which exists between subbands coefficients. Intra-band, inter-band and inter-channel dependencies have to be considered. Let us detail each of these dependencies for the three color components.

\subsubsection{Intra-band dependency}

Intra-band dependence refers to the spatial neighborhood dependence between coefficients of the same wavelet subband. It corresponds to the spatial structure of the studied subband. Let $s$ be the location of a reference coefficient from the $k^{\text {th }}$ subband of a given color channel. If we consider the luminance channel for example, let $m$ be the size of the chosen neighborhood. The collection of the reference coefficient neighbors is specified by $l_{s}^{(k)}=\left(l_{1, s}^{(k)}, \cdots, l_{m, s}^{(k)}\right)$.

Then, considering all the subbands coefficient neighbors, the dataset to be observed is:

$$
l_{\text {intra }}=\left[l_{1}^{(k)}, \cdots, l_{s}^{(k)}, \cdots, l_{P}^{(k)}\right]^{T}
$$

$l_{\text {intra }}$ is a $P$ by $m$ matrix, where $P$ is the size of the wavelet subband. If we consider, the first chrominance channels, intra-band dependency for a given subband $k$ is studied by observing the following dataset:

$$
c r_{1_{\text {intra }}}=\left[c r_{1}{ }_{1}^{(k)}, \cdots, c r_{1}^{(k)}, \cdots, c r_{1}{ }_{P}^{(k)}\right]^{T}
$$

For the second chrominance channel, we have:

$$
c r_{2_{\text {intra }}}=\left[c r_{2}{ }^{(k)}, \cdots, c r_{2}{ }_{s}^{(k)}, \cdots, c r_{2}{ }_{P}^{(k)}\right]^{T}
$$

\subsubsection{Inter-band dependency}

To model the inter-band dependence, we consider subbands in different orientations for the same color channel. Thus for luminance inter-band we observe a dataset represented by a $P$ by $K$ matrix, where $K$ is the total number of subbands:

$$
l_{\text {interbd }}=\left[l^{(1)} \cdots l^{(K)}\right]
$$

for chrominance inter-band:

$$
\begin{aligned}
& c r_{1_{\text {interbd }}}=\left[c r_{1}^{(1)} \cdots c r_{1}^{(K)}\right] \\
& c r_{2_{\text {interbd }}}=\left[c r_{2}^{(1)} \cdots c r_{2}^{(K)}\right]
\end{aligned}
$$

we note that $l^{(k)}, c r_{1}^{(k)}$ and $c r_{2}^{(k)}$ are respectively the column vectors containing all coefficients of the $k^{\text {th }}$ subband of luminance, first and second chrominance channels.

\subsubsection{Inter-channel dependency}

It refers to the dependence between coefficients of subbands from different color channel, considering a fixed orientation $k$. The observation matrix is as follows:

$$
D_{\text {interch }}=\left[\begin{array}{lll}
l^{(k)} & c r_{1}^{(k)} & c r_{2}^{(k)}
\end{array}\right]
$$

\subsubsection{Full dependence}

Considering all the aforementioned dependencies leads to a full dataset that considers intra-band, inter-band and inter-channel dependencies jointly. It is represented by the following matrix of size $P$ by $(3 \times K \times m)$ :

$$
\begin{aligned}
D= & {\left[l_{1}^{(1)}, \cdots, l_{P}^{(1)}, \cdots, l_{1}^{(K)}, \cdots, l_{P}^{(K)}, c r_{11}^{(1)}, \cdots, c r_{1}{ }_{P}^{(1)}\right.} \\
& \cdots, c r_{11}^{(K)}, \cdots, c r_{1}^{(K)}, c r_{21}^{(1)}, \cdots, c r_{2}^{(1)}, \cdots, c r_{21}^{(K)}\left({ }^{(K)}\right) \\
& \left.\cdots, c r_{2}{ }_{P}^{(K)}\right]^{T}
\end{aligned}
$$

Consideration of this matrix means, first, that all the three components carry the spatial structure information (intraband). Second, that dependence exists between subbands of all orientations for each channel (inter-band), and third, that the representation exhibits dependence among the three color channels (inter-channel).

\subsection{Multi-model approach}

\subsubsection{Experimental analysis of dependence}

Contrary to the RGB representation, LC color spaces offer separability between luminance and chrominance. At first glance, this indicates that the inter-channel dependence have not to be considered between luminance and chrominance. A more precise study of dependence is done according to the correlation coefficients. Table 1 shows degrees of dependence when textures are represented in Lab color space. This is obtained by averaging the Pearson, Kendall, Spearman and the mutual information coefficients on all subbands of 40 color textures from the Vistex database (Vistex). We recall that mutual information is a measure of the statistical dependence between two variables. If we suppose that $X$ and $Y$ are two random variables, $f(x)$ and $f(y)$ the marginal probability distribution functions of $X$ and $Y$ respectively, and $f(x, y)$ the joint probability distribution function of $X$ and $Y$. The mutual information between $X$ and $Y$ is then:

$$
I(X, Y)=\sum_{x, y} f(x, y) \log \frac{f(x, y)}{f(x) f(y)}
$$

We deduce from these results that intra-band dependence have to be considered only for luminance, and that interchannel dependence exists only between chrominance components. They also show that we can restrict the study of inter-band dependence to the luminance component.

\subsubsection{Multi-model formulation}

Our contribution here is to exploit the independence between luminance and chrominance. So we propose a multimodel based characterization that considers the different natures of these information ((Qazi et al. 2011)). Two joint and independent models $M_{L}$ and $M_{C_{r}}$ are used to respectively characterize the luminance information with 
Table 1: Averaging measure of dependence on the Vistex database, using Pearson, Kendall, Spearman and mutual information for 40 color textures from the Vistex database in the Lab color space.

\begin{tabular}{cccc|ccc|ccc} 
& \multicolumn{3}{c|}{ Intra-band } & \multicolumn{3}{c|}{ Inter-band } & \multicolumn{3}{c}{ Inter-channel } \\
\hline & $l$ & $c r_{1}$ & $c r_{2}$ & $l$ & $c r_{1}$ & $c r_{2}$ & $\left\{l, c r_{1}\right\}$ & $\left\{l, c r_{2}\right\}$ & $\left\{c r_{1}, c r_{2}\right\}$ \\
\hline Pearson & 0.47 & 0.041 & 0.0372 & 0.33 & 0.037 & 0.041 & 0.003 & 0.003 & 1 \\
Kendall & 0.45 & 0.038 & 0.037 & 0.28 & 0.037 & 0.038 & 0.002 & 0.002 & 1 \\
Spearman & 0.32 & 0.026 & 0.025 & 0.25 & 0.025 & 0.026 & 0.001 & 0.001 & 1 \\
MI & 4.46 & 1.56 & 1.56 & 4.20 & 1.03 & 1.04 & 0.74 & 0.75 & 5.88 \\
\hline
\end{tabular}

spatial and inter-band dependence considering observations of a vector $\bar{l}_{s}$ (see subsection 2.4.1) and the chrominance one devoted to inter-channel dependence by observing realizations of a vector $\mathrm{cr}_{s}$ (see subsection 2.4.2):

$$
p\left(x_{T}, \theta\right)=p\left(x_{T} \mid\left\{M_{L}, M_{C_{r}}\right\}\right) \propto p\left(\bar{l}_{s}, \theta_{L}\right) \prod_{k=1}^{K} p\left(c r_{s}^{(k)} \mid \theta_{c_{r}}\right)
$$

where $\theta_{L}$ and $\theta_{c_{r}}$ are the vectors of parameters of luminance and chrominance models, respectively. The multimodeling permits to reduce the dimension of the observed dependence dataset $D$ (subsection 2.1).

\subsection{Copula based multivariate models}

Different multivariate models can be used for defining $M_{L}$ and $M_{C_{r}}$, such as the multivariate generalized Gaussian (MGGD) (Verdoolaege et al. 2012), the multivariate Bessel K forms (Boubchir et al. 2010), the spherically invariant random vector (SIRV) (Lasmar et al. 2010). Those models have been proposed to characterize different kinds of dependencies such as the intra-band in the case of SIRV and the inter-channel in the case of MGGD. Each of them is dedicated to a specific dependency rule, either for intra-band or inter-channel but never taking account of both together. Obviously, extending each model simultaneously to the both dependencies is possible but the equivalent model exhibits the main drawback the common definition of the leptokurtic and platykurtic for the whole set components of the multivariate model. If we suppose to have a set of dependent variables $T=\left[t_{1}, \cdots, t_{n}\right]$, with the MGGD or SIRV multivariate modeling, each marginal $t_{i}$ has finally a similar shape value. This behavior is confirmed in the study of (Rangaswamy et al. 2002), the characterization of one marginal is sufficient to determine the shape of the whole distribution of a SIRV. Table 2 show averages of scale and shape parameters supposing that subbands of the three components are described by the same marginal, Weibull or Gamma for example. It is clear from the table that the marginal modeling of luminance and chrominance exhibits different shape and scale parameters in the case of Lab color space, contrary to RGB. In the HSV color space, we observe that parameters are different even between the chrominance components $\mathrm{H}$ and $\mathrm{S}$.

The copula theory allows the joint modeling by separating the dependence structure from the marginal modeling. It is thus easy to merge different marginal laws into the
Table 2: Average of parameters (considering wavelet subbands for 40 color textures from Vistex database), supposing that subbands of the three color components are described by the same marginale (Weibull or Gamma) in the case of Lab, HSv and RGB color spaces

\begin{tabular}{ccccc}
\hline & \multicolumn{2}{c}{ Weibull } & \multicolumn{2}{c}{ Gamma } \\
\hline & scale & shape & scale & shape \\
\hline $\mathrm{L}$ & 11.47 & 1.54 & 4.97 & 2.18 \\
$\mathrm{a}$ & 3.21 & 1.63 & 1.26 & 2.41 \\
$\mathrm{~b}$ & 2.20 & 1.62 & 0.92 & 2.39 \\
\hline \hline $\mathrm{H}$ & 26.83 & 1.08 & 27.15 & 1.29 \\
$\mathrm{~S}$ & 0.12 & 1.53 & 0.05 & 2.16 \\
$\mathrm{~V}$ & 0.0081 & 1.52 & 0.03 & 2.21 \\
\hline \hline $\mathrm{R}$ & 31.51 & 1.53 & 13.83 & 2.16 \\
$\mathrm{G}$ & 29.91 & 1.52 & 13.33 & 2.13 \\
$\mathrm{~B}$ & 31.25 & 1.52 & 13.85 & 2.14 \\
\hline
\end{tabular}

same multivariate pdf. (Nelsen 2006) defined the copula as a multivariate uniform distribution used to construct multivariate models. Given that $F$ is a $d$-dimensional cumulative distribution function (cdf) with continuous marginals $F_{1}, \ldots, F_{d}$, the Sklar's theorem (Sklar et al. 1959) shows that there exist a unique copula $C$ such that:

$$
F\left(x_{1}, \cdots, x_{d}\right)=C\left(F_{1}\left(x_{1}\right), \cdots, F_{d}\left(x_{d}\right)\right)
$$

Further, if $C$ is continuous and differentiable, the copula density $(c)$ is given by:

$$
c\left(u_{1}, \ldots, u_{d}\right)=\frac{\partial^{d} C\left(u_{1}, \ldots, u_{d}\right)}{\partial u_{1} \ldots \partial u_{d}}
$$

The joint pdf is uniquely deduced from the marginals and the copula density as follows:

$$
f\left(x_{1}, \ldots, x_{d}\right)=c\left(F_{1}\left(x_{1}\right), \ldots, F_{d}\left(x_{d}\right)\right) \prod_{i=1}^{d} f_{i}\left(x_{i}\right)
$$

where $f_{i}, \quad i=1, \cdots, d$, represent the marginal pdfs that can follow different laws.

Estimation of the copula parameters is done using the canonical maximum likelihood (CML) (Durrleman et al. 1959) method. This is a pseudo parametric estimation method contrary to the fully parametric method, termed the inference from marginals 
(IFM) (Joe. 1997). The CML method consists in transforming the data $\left(x_{1}, \ldots, x_{d}\right)$ into uniform data $\left(\hat{u}_{1}, \ldots, \hat{u}_{d}\right)$ using the empirical distributions (empirical cdfs) and then estimates the copula parameters considering all the observations as:

$$
\hat{\Sigma}=\underset{\Sigma}{\operatorname{argmax}} \sum_{i=1}^{n} \log c\left(\hat{u}_{i 1}, \ldots, \hat{u}_{i d} ; \Sigma\right)
$$

where $n$ denotes the number of observations, and $\Sigma$ represents the correlation matrix.

For marginal parameters, the estimation is done using the conventional maximum likelihood method. Experiments reveal that the CML estimator is the best since the method does not rely on parametric assumption about marginals. This means that less pertinent choice of marginals do not affect estimation of the copula parameters.

For texture modeling, we repose on copulas to construct the models $M_{L}$ and $M_{C_{r}}$. We select the Gaussian copula for which the density is defined by:

$$
c_{\phi}\left(u_{1}, \cdots, u_{d}\right)=\frac{1}{|\Sigma|^{1 / 2}} \exp \left[-\frac{1}{2} \vartheta^{T}\left(\Sigma^{-1}-I\right) \vartheta\right]
$$

with $\vartheta^{T}=\left(\phi^{-1}\left(u_{1}\right), \cdots, \phi^{-1}\left(u_{d}\right)\right), \phi$ denotes the cdf of the normalized Gaussian density, $\Sigma$ the correlation matrix, and $I$ the $d$-dimensional identity matrix.

The choice of the Gaussian copula is justified by:

- the dependence representation, which is based on the well known correlation coefficient.

- the existence of closed form of Rao distance for the Gaussian copula which allow us to derive a closed form of Rao distance for the joint model as a similarity measure (subsection 3.1).

\section{4. $M_{L}$ and $M_{C_{r}}$ models}

Considering the full dependence dataset $D$ (see subsection 2.1.4) leads to high parametrization and thus to very cumbersome estimation of parameters. To alleviate this complexity, we use the proposition made by (Qazi et al. 2011) about dependencies between coefficient subbands when color textures are represented in LC color spaces. The proposition consists in a multi-modeling approach to reduce the dimensionality of the observed dataset.

\subsubsection{Multivariate spatial model for luminance $\left(M_{L}\right)$}

As a first step for reducing dimension of the full dataset $D$, the spatial structure along intra- and inter-band dependence are studied only for luminance via the model $M_{L}$. For a subband coefficient at the position $s$, intra inter-band dependence is represented by the vector $\bar{l}_{s}=\left(l_{1, s}, \cdots, l_{m, s}, \cdots, l_{d, s}\right)$, where $d=m \times K, m$ is the neighborhood size and $K$ is the number of wavelet subbands. Considering all positions at the luminance subbands, the dataset to be modeled is a $P$ by $d$ matrix of realizations of the vector $\bar{l}$ :

$$
L=\left[\begin{array}{c}
\bar{l}_{1} \\
\bar{l}_{2} \\
\vdots \\
\bar{l}_{s} \\
\vdots \\
\bar{l}_{P}
\end{array}\right]
$$

where $P$ is the size of a wavelet subband. The observation matrix is:

$$
L=\left[\begin{array}{ccccc}
l_{1,1} & \cdots & l_{m, 1} & \cdots & l_{d, 1} \\
\vdots & & \vdots & & \vdots \\
l_{1, s} & \cdots & l_{m, s} & \cdots & l_{d, s} \\
\vdots & & \vdots & & \vdots \\
l_{1, P} & \cdots & l_{m, P} & \cdots & l_{d, P}
\end{array}\right]
$$

Based on the Gaussian copula, the pdf of the model $M_{L}$ is defined by:

$$
f_{M_{L}}\left(\bar{l}_{s} ; \theta\right)=\frac{1}{\left|\Sigma_{L}\right|^{1 / 2}} \exp \left[-\frac{1}{2} \vartheta_{s}^{T}\left(\Sigma_{L}^{-1}-I\right) \vartheta_{s}\right] \prod_{i=1}^{d} f_{s}\left(\bar{l}_{i, s}\right)
$$

where $\theta=\left(w_{1}, w_{2}, \ldots, w_{d} ; \Sigma_{L}\right)$ is the vector of hyperparameters, $w_{s}=\left\{w_{s j}\right\}_{j \in \mathcal{N}}, s=1, \cdots, d$ is the vector of parameters for the marginal $f_{s}$ and $\Sigma_{L}$ is the covariance matrix of the Gaussian copula.

In the case of Gaussian copula, the CML estimator of covariance matrix is the sample correlation matrix of Gaussian observations $\vartheta_{1}, \cdots, \vartheta_{P}$ :

$$
\hat{\Sigma}_{L}=\frac{1}{P} \sum_{s=1}^{P} \vartheta_{s} \vartheta_{s}^{T}
$$

with $\vartheta_{s}=\left(\vartheta_{1, s}, \cdots, \vartheta_{d, s}\right)$ is obtained by transforming the observation $\bar{l}_{s}=\left(l_{1, s}, \cdots, l_{d, s}\right)$ from the observation matrix $L$ by $\vartheta_{i, s}=\phi^{-1}\left(F_{i}\left(l_{i, s}\right)\right), i=1, \cdots, d$ and $F_{s}$ is the empirical marginal cdf.

\subsubsection{Circular/Linear bivariate model for chrominance $\left(M_{C_{r}}\right)$}

We consider the inter-channel dependence only between chrominance component subband represented by the vector $c r=\left(c r_{1}^{(k)}, c r_{2}^{(k)}\right)$. Thus, considering all coefficients of the chrominance subbands, the dataset to be modeled by $M_{C_{r}}$ is a $P$ by 2 matrix:

$$
c r=\left[\begin{array}{cc}
c r_{1,1} & c r_{2,1} \\
\vdots & \vdots \\
\vdots & \vdots \\
c r_{1, P} & c r_{1, P}
\end{array}\right]
$$

$M_{C_{r}}$ is a bivariate model defined reposing on the Gaussian copula:

$f_{M_{C_{r}}}\left(c r_{s}^{(k)} ; \theta\right)=\frac{1}{\left|\Sigma_{C_{r}}\right|^{1 / 2}} \exp \left[-\frac{1}{2} \vartheta_{s}^{T}\left(\Sigma_{C_{r}}^{-1}-I\right) \vartheta_{s}\right] \prod_{i=1}^{2} f_{i}\left(c r_{i}\right)$ 
where $\theta=\left(w_{1}, w_{2} ; \Sigma_{C_{r}}\right), w_{i}=\left\{w_{i j}\right\}_{j \in \mathfrak{\aleph}}$. The index $i \in\{1,2\}$ is the vector of parameters for the marginal $f_{i}$, and $\Sigma_{C_{r}}$ the covariance matrix. The CML estimator of the covariance matrix is as follows:

$$
\hat{\Sigma}_{C_{r}}=\frac{1}{P} \sum_{s=1}^{P} \vartheta_{s} \vartheta_{s}^{T}
$$

with $\vartheta_{s}=\left(\vartheta_{1, s}, \vartheta_{2, s}\right)$ is obtained by transforming the observation $c r_{s}=\left(c r_{1, s}, c r_{2, s}\right)$ from the observation matrix $c r$ by $\vartheta_{i, s}=\phi^{-1}\left(F_{i}\left(c r_{i, s}\right)\right), i=1,2$ and $F_{s}$ is the empirical marginal cdf.

The model is called circular/linear because it offers the possibility of using different marginals $f_{i}$ to come up with a bivariate circular/linear, circular/circular or linear/linear model for chrominance channels depending on the nature of the used color space.

\subsubsection{Texture joint modeling}

Taking into account the previous sub-models, respectively $M_{L}$ and $M_{C_{r}}$ termed probabilistic because they describe probability distributions over sub-band coefficients of the texture, let us consider a stationary observed texture, we have:

$$
p\left(x_{T} \mid\left\{M_{L}, M_{C_{r}}\right\}\right) \propto p\left(\bar{l}_{s} \mid w_{s}, \Sigma_{L}\right) \prod_{k=1}^{K} p\left(c r_{s}^{(k)} \mid w_{c r_{1}}^{(k)}, w_{c r_{2}}^{(k)}, \Sigma_{c_{r}}^{(k)}\right)
$$

which can be written in a more compact parametric form as:

$$
p\left(x_{T} \mid\left\{M_{L}, M_{C_{r}}\right\}\right) \propto p\left(x_{T} \mid \theta_{s}\right)
$$

with $\theta_{s}=\left(w_{s}, \Sigma_{L},\left\{w_{c r_{1}}^{(k)}, w_{c r_{2}}^{(k)} \Sigma_{c_{r}}^{(k)}\right\}_{k=1, \cdots, K}\right)$

\section{Classification}

In the framework of stochastic parametric classifier, we consider supervised Bayes classifier to assign the most probable class to a textured image. If we consider the equiprobability of each class, the Bayes classifier consists in finding the maximum of the likelihood such as:

$$
\hat{b}=\underset{b \in\{1,2, \ldots, B\}}{\operatorname{argmax}}\left[p\left(x_{T}, \theta_{b}\right)\right]
$$

where $\theta_{b}$ is the vector of parameters associated to the class $b$. The vector $\theta_{b}$ is estimated during the learning step from the set of texture samples representing the class $b$. Considering equation 22, the decision of the classifier is provided by the numerical evaluation of the likelihood which is time consuming because of evaluating equation 21 from the three color channel data. In order to avoid this expensive step, a direct comparison between parametric vectors, i.e. one representing the class and the other the texture query $x_{T}$, is expected. Thus, the class assignment is obtained from a similarity measure. To do this, the Softmax approach or normalized exponential function (Bishop 2006) is selected.

$$
\underset{b \in\{1,2, \ldots, B\}}{\operatorname{argmax}} \frac{\exp \left(a_{b}\right)}{\sum_{j=1}^{B} \exp \left(a_{j}\right)}
$$

with $a_{b}=\ln \left[\frac{1}{1+L_{i}\left(x_{T}, x_{b}\right)}\right]$.

The advantage of using the softmax principal is the smoothed version of the maximum, since if $a_{b} \gg a_{j}$ for all $j \neq b$, then $p\left(C_{b} \mid x_{T}\right) \simeq 1$ and $p\left(C_{j} \mid x_{T}\right) \simeq 0$. The proposed approach is thus based on the selection of a similarity measure, i.e $L_{i}\left(x_{T}, x_{b}\right)$ exhibiting a closed form in terms of model parameters. Various probabilistic measures of similarity propose this kind of properties such as the KullbackLeibler or Hellinger measures. All these possibilities have been unified in the seminal theory of the information geometry. This consists in considering that the parametric model of finite dimension forms a sub-manifold characterized by a smooth Riemannian space. Some of these measures are divergences such as Kullback-Leibler. A divergence is a non-symmetric measure and can be not respecting the triangle inequality. Some others such as Bhattacharya, Hellinger or others are really some distances. Considering the properties of the Riemmanian manifold, the well-founded distance associated to this type of manifold is the geodesic or Rao distance (Atkinson et al. 1981). The main drawback of the geodesic distance is that a closed form for probabilistic model is rarely available. This is the reason for which the Kullback-Leibler divergence is usualy used in place to the geodesic distance. However, the true distance according to the Riemannian space is the geodesic distance.

In the next subsection, we derive for our context a closed form for the geodesic distance for the Copula modeling.

\subsection{Proposed Rao distance as similarity measure}

For a probability density $f(x ; \theta)$ with $\theta=\left(\theta^{1}, \cdots, \theta^{N}\right)$ a vector of parameters, the Rao distance is a Riemannian metric defined by the fisher information matrix as:

$$
d s^{2}=\sum_{p, q=1}^{N} g_{p q}(\theta) d \theta^{i} d \theta^{j}
$$

where $g_{p q}$ represent the Fisher matrix elements:

$$
g_{p q}(\theta)=-E\left[\frac{\partial^{2}}{\partial \theta^{p} \theta^{q}} \log f(x ; \theta)\right]
$$

Let $f$ be a Gaussian copula based joint pdf $\left(M_{L}\right.$ or $\left.M_{C_{r}}\right), \theta=\left\{w_{i}, \Sigma\right\}$ is the vector of hyper-parameters of $f$, $w_{i}=\left\{w_{i j}\right\}_{j \in \mathcal{N}}$ is the vector of parameters for the marginal $f_{i}, i=1, \cdots, d$ (for example if the marginal is a Weibull we have $w_{i}=\left\{\alpha_{i}, \beta_{i}\right\}$ representing shape and scale parameters), $d$ is the length of the vector of one observation and $\Sigma$ represents the covariance matrix of the Gaussian 
copula. By applying equations 13 and 15 in equation 25 we have:

$g_{p q}(\theta)=-E\left[\frac{\partial^{2}}{\partial \theta^{p} \theta^{q}}\left(\log c_{\Phi}(u ; \Sigma)+\log \prod_{i=1}^{d} f_{i}\left(x_{i} ; w_{i}\right)\right)\right]$

where $u=\left(u_{1}, \ldots, u_{d}\right)$ represents the vector of uniform coefficients that are obtained by transforming the wavelet coefficients $\left(x_{1}, \ldots, x_{d}\right)$ using their cdfs $\left(u_{i}=F^{-1}\left(x_{i}, w_{i}\right)\right)$ or using their empirical cdfs (see MATLAB's routine "ecdf" ) $\left(u_{i}=e F^{-1}\left(x_{i}\right)\right)$. In this latter case, no assumptions are made on the parametric form of the marginal distributions. The copula parameters are then independent from any marginal parameter $w_{i j}$ :

$$
\frac{\partial}{\partial w_{i j}} c_{\Phi}(u ; \Sigma)=0
$$

so then, $g_{w_{i j} \Sigma}(\theta)=g_{\Sigma w_{i j}}(\theta)=0, \quad i=1 \cdots d, \quad j \in \aleph$ Thus,

$$
g_{\Sigma \Sigma}(\theta)=-E\left[\frac{\partial^{2}}{\partial \Sigma \partial \Sigma} \log c_{\Phi}(u ; \Sigma)\right]
$$

and

$$
g_{\mu \nu}(\theta)=-E\left[\frac{\partial^{2}}{\partial \mu \partial \nu} \log \prod_{i=1}^{d} f_{i}\left(x_{i} ; w_{i}\right)\right]
$$

with $\mu, \nu \in\left\{w_{i j}\right\}, \quad i=1 \cdots d, \quad j \in \aleph$

$$
\begin{aligned}
& g_{\mu \nu}(\theta)=-E\left[\frac{\partial^{2}}{\partial \mu \partial \nu} \sum_{i=1}^{d} \log f_{i}\left(x_{i} ; w_{i}\right)\right] \\
& =-E\left[\sum_{i=1}^{d} \frac{\partial^{2}}{\partial \mu \partial \nu} \log f_{i}\left(x_{i} ; w_{i}\right)\right] \\
& =\sum_{i=1}^{d}-E\left[\frac{\partial^{2}}{\partial \mu \partial \nu} \log f_{i}\left(x_{i} ; w_{i}\right)\right]
\end{aligned}
$$

Thus, from (12):

$$
\begin{array}{r}
d s^{2}=g_{\Sigma \Sigma} d \Sigma d \Sigma+\sum_{i=1}^{d} \sum_{\mu, \nu} g_{\mu \nu} \dot{\mu} \dot{\nu} d t \\
=d s_{\text {Gauss }}^{2}+\sum_{i=1}^{d} d s_{\text {Margins }}^{2}
\end{array}
$$

Hence, given two copula based probability distributions $f^{(1)}\left(x ; \theta_{1}\right)$ and $f^{(2)}\left(x ; \theta_{2}\right)$ with $\theta_{1}=\left(w_{1}^{(1)}, \cdots, w_{d}^{(1)}, \Sigma_{1}\right)$ and $\theta_{2}=\left(w_{1}^{(2)}, \cdots, w_{d}^{(2)}, \Sigma_{2}\right)$, we can compute the Rao geodesic distance as the sum of the Rao distances of the Gaussian distribution and the Rao distances between marginals (El Maliani et al. 2011):

$$
L^{2}=\int_{\theta_{1}}^{\theta_{2}} d s^{2}=\int_{\theta_{1}}^{\theta_{2}} d s_{\text {Gauss }}^{2}+\sum_{i=1}^{d} \int_{\theta_{1}}^{\theta_{2}} d s_{\text {Margins }}^{2}
$$

$$
\begin{aligned}
& L^{2}=L_{\text {Gauss }}^{2}\left(f^{(1)}\left(x ; \Sigma_{1}\right) \| f^{(2)}\left(x ; \Sigma_{2}\right)\right)+ \\
& \sum_{i=1}^{d} L_{\text {Margins }}^{2}\left(f_{i}^{(1)}\left(x ; w_{i}^{(1)}\right) \| f_{i}^{(2)}\left(x ; w_{i}^{(2)}\right)\right)
\end{aligned}
$$

that is:

$$
\begin{aligned}
& L^{2}=\frac{1}{2} \sum_{i=1}^{d}\left(\ln \lambda^{i}\right)^{2}+ \\
& \sum_{i=1}^{d} L_{\text {Margins }}^{2}\left(f_{i}^{(1)}\left(x ; w_{i}^{(1)}\right) \| f_{i}^{(2)}\left(x ; w_{i}^{(2)}\right)\right)
\end{aligned}
$$

where $\lambda^{i}, i=1, \ldots, d$ represents the eigenvalues of $\Sigma_{1}^{-1} \Sigma_{2}$.

\section{EXPERIMENTAL RESULTS}

The experimental section is concerned with five major issues:

1. Choice of the multi-model for color texture: for this, we investigate a variety of models for different color spaces.

2. Choice of the LC color space: we compare performances between two sub-families of LC color spaces, namely the perceptually uniform color spaces (represented by Lab), and the perceptual color spaces (represented by $\mathrm{HSV}$ ).

3. Comparison with existing approaches: we evaluate performances of our approach in comparison with existing uni-modeling approaches in LC color spaces (Qazi et al. 2010; Qazi et al. 2011) and in RGB color space (Verdoolaege et al. 2012; Kwitt et al. 2011).

4. Runtime: we address the runtime issue by comparing the proposed Rao distance based similarity measure with the Monte-carlo based Kullback-leibler divergence between copula based pdfs.

5. Curse of dimensionality: we compare our approach with the full feature vector based approach (subsection 2.1).

\subsection{Experiments versus data diversity}

In order to conduct representative experiments, we use different databases and various configurations of the experimental protocol leading to a large view of the proposed multi-model performance. The conventional Vistex database (Verdoolaege et al. 2012; Kwitt et al. 2011; Do et al. 2002) is used considering two sizes of samples respectively $32 \times 32$ and $128 \times 128$. Experiments are also conducted on the Outex, ALOT and Stex databases which are more challenging color texture database, since the color and texture information are not easily distinguishable. We consider the following scenarios: 
- First scenario, i.e. DB1, addressing a set of 24 textured images of size $512 \times 512$ from the Vistex database (Vistex). The protocol follows the work of (Permuter et al. 2006) and (Qazi et al. 2010). Each image was divided into subimages of size $32 \times 32$ pixels resulting of a large database of 6144 small textures. We consider 96 image from the resulting 256 subimages as the training set, while the remaining 160 subimages are considered as the test set. This dataset is used in order to evaluate robustness of our model even if very local spatial structures are considered.

- Second scenario, i.e. DB2, addressing a set of 54 textured images of size $512 \times 512$ from the Vistex database. The protocol follows the work of (Do et al. 2002). Each image was divided into subimages of size $128 \times 128$ pixels. This dataset is available on the Outex web site (Ojala et al.; Outex) as test suit Contrib_TC_00006. For each texture, subimages are considered to form a checkerboard. The white half of subimages is then considered as the training set and the black half is used as the testing set. Hence, the training procedure will account for non uniformity of the original images.

- Third scenario, i.e. DB3, addressing a set of 68 textured images of size $746 \times 538$ from the Outex database. This dataset is also available on the Outex web site as Outex_TC_00013. Each image was divided into 20 subimages of size $128 \times 128$ pixels. Training and test sets are obtained as in DB2.

- Fourth scenario, i.e. DB4, addressing large set of classes from ALOT (ALOT) database. 250 images of size $384 \times 256$ from ALOT databse are considered, then each image is split into 16 non-overlapping subimages resulting on two large databases of 4000 images of size $96 \times 64$. These images have been captured uder different viewing angles and illumination. Here again training and test sets are obtained as in DB2.

- Fifth scenario, i.e. DB5, addressing large set of classes from Stex (STex) database. 476 textures from STex of size $512 \times 512$ are considered. Then, each image is split into 16 non-overlapping subimages resulting on two large databases of 7616 images of size $128 \times 128$. Here again training and test sets are obtained as in DB2.

Every color component of each subimage was normalized by subtracting its mean and dividing by its standard deviation, and then decomposed using a 2-scale dual-tree complex wavelet transform (DTCWT) (Kingsbury 1998) with a Q-shift $(14,14)$ tap filter. In addition to its rich directional analysis, shift invariance and low redundancy properties, the DTCWT was chosen for its reduced computational time.

\subsection{Quantitative evaluation of performance}

Evaluation of the classification performance of each approach consists on two criteria, namely the percentage of classification and the precision. We recall that for each class of textures:

- True positives (TP): refers to the number of subimages that have been successfully returned by the classifier as belonging to a given class.

- False positives (FP): is the number of subimages that have been wrongly classified as belonging to a given class.

- False negatives (FN): is the number of subimages that are classified as not belonging to a given class while, in fact, they belong to this class of textures.

- True negatives $(\mathrm{TN})$ : is the number of subimages that are correctly classified as not belonging to a given class.

The percentage of classification (or Sensitivity) is the proportion of textures which were well labeled by the classifier. It represents the ability of the classifier in correct positive assignment of a texture to its real class. A $100 \%$ percentage means that the classifier makes no false negative labeling $(\mathrm{FN}=0)$. The Percentage classification is given as follows:

$$
\mathrm{Sn}=\frac{\mathrm{TP}}{\mathrm{TP}+\mathrm{FN}} \times 100 \%
$$

However, theoretically, a system that always returns positive assignment (even wrongly) will achieve $100 \%$ percentage classification. The problem with percentage classification is that it tells nothing about textures that were wrongly labeled as belonging to a given class (FP assignments). For this, we take into account also the precision to evaluate the classifier performance.

The precision criterion indicates the ability of the classifier to detect true class membership. The precision is given by:

$$
\mathrm{Sp}=\frac{\mathrm{TP}}{\mathrm{TP}+\mathrm{FP}}
$$

A classifier with very high precision makes no mistakes in assigning a texture to its class $(\mathrm{FP}=0)$.

\subsection{Results and discussions}

One of the advantages of the proposed multi-modeling approach is the flexibility in term of modeling textures from different LC color spaces. The joint models $M_{L}$ and $M_{C_{r}}$ enable us to consider different parametric modeling for marginals between luminance and chrominance channels. As already mentioned, when chrominance components are defined in a cylindrical space, the hue represents a circular component and the saturation is the axe of the cylinder. We can then benefit from the advantage of copulas in merging different marginals in the same joint model 
Table 3: Notations, descriptions and pdf expressions for the used models

\begin{tabular}{|c|c|c|}
\hline Model & Description & pdf \\
\hline CopMGam & $\begin{array}{l}\text { Gaussian copula based multivariate Gamma (all } \\
\text { marginals are Gamma) }\end{array}$ & $\begin{array}{l}f(x, \theta)=\frac{1}{|\Sigma|^{1 / 2}} \exp \left[-\frac{1}{2} \vartheta^{T}\left(\Sigma^{-1}-I\right) \vartheta\right] \times \\
\left(\frac{\beta^{-\alpha}}{\Gamma(\alpha)}\right)^{d} \prod_{i=1}^{d} x_{i}^{\alpha-1} \exp -\sum_{i=1}^{d}\left(\frac{x_{i}}{\beta}\right), \quad \theta= \\
(\alpha, \beta, \Sigma)\end{array}$ \\
\hline CopMWbl & $\begin{array}{l}\text { Gaussian copula based multivariate Weibull (all } \\
\text { marginals are Weibull) }\end{array}$ & $\begin{array}{l}f(x, \theta)=\frac{1}{|\Sigma|^{1 / 2}} \exp \left[-\frac{1}{2} \vartheta^{T}\left(\Sigma^{-1}-I\right) \vartheta\right] \times \\
\left(\frac{\tau}{\lambda}\right)^{d} \prod_{i=1}^{d} x_{i}^{\tau-1} \exp -\sum_{i=1}^{d}\left(\frac{x_{i}}{\lambda}\right)^{\tau}, \quad \theta= \\
(\tau, \lambda, \Sigma)\end{array}$ \\
\hline$\{$ Gamma, Weibull $\}$ & $\begin{array}{l}\text { Gaussian copula based bivariate model with } \\
\text { Gamma as the fisrt marginal and Weibull as the } \\
\text { second marginal }\end{array}$ & $\begin{array}{l}f(x, \theta)=\frac{1}{|\Sigma|^{1 / 2}} \exp \left[-\frac{1}{2} \vartheta^{T}\left(\Sigma^{-1}-I\right) \vartheta\right] \times \\
\frac{\tau \beta^{-\alpha} x_{2}^{\alpha-1}}{\lambda \Gamma(\alpha)}\left(\frac{x_{1}}{\lambda}\right)^{\tau-1} \times \exp \left[-\left(\frac{x_{1}}{\lambda}\right)^{\tau}-\frac{x_{2}}{\beta}\right], \theta= \\
(\alpha, \beta, \tau, \lambda, \Sigma)\end{array}$ \\
\hline$\{$ vonMises, Weibull $\}$ & $\begin{array}{l}\text { Gaussian copula based circular/linear bivariate } \\
\text { model with vonMises as the fisrt marginal and } \\
\text { Weibull as the second marginal }\end{array}$ & $\begin{array}{l}f(x, \theta)=\frac{1}{|\Sigma|^{1 / 2}} \exp \left[-\frac{1}{2} \vartheta^{T}\left(\Sigma^{-1}-I\right) \vartheta\right] \times \\
\frac{\tau}{2 \pi \lambda I_{0}(\nu)}\left(\frac{x_{2}}{\lambda}\right)^{\tau-1} \exp \left[\nu \cos \left(x_{1}-\mu\right)-\left(\frac{x_{2}}{\lambda}\right)^{\tau}\right], \theta= \\
(\mu, \nu, \tau, \lambda, \Sigma)\end{array}$ \\
\hline$\{$ vonMises, Gamma $\}$ & $\begin{array}{l}\text { Gaussian copula based circular/linear bivariate } \\
\text { model with vonMises as the fisrt marginal and } \\
\text { Gamma as the second marginal }\end{array}$ & $\begin{array}{l}f(x, \theta)=\frac{1}{|\Sigma|^{1 / 2}} \exp \left[-\frac{1}{2} \vartheta^{T}\left(\Sigma^{-1}-I\right) \vartheta\right] \times \\
\frac{\beta^{-\alpha}}{2 \pi \Gamma(\alpha) I_{0}(\nu)}\left(\frac{x_{2}}{\beta}\right)^{\alpha-1} \exp \left[\nu \cos \left(x_{1}-\mu\right)-\right. \\
\left.\left(\frac{x_{2}}{\beta}\right)^{\alpha}\right], \theta=(\mu, \nu, \alpha, \beta, \Sigma)\end{array}$ \\
\hline
\end{tabular}

Table 4: Performances of different versions of the multi-model in Lab and HSV color spaces.

\begin{tabular}{ccccccccccc} 
& \multicolumn{2}{c}{ DB1 } & \multicolumn{2}{c}{ DB2 } & \multicolumn{2}{c}{ DB3 } & \multicolumn{2}{c}{ DB4 } & \multicolumn{2}{c}{ DB5 } \\
\hline & Sn & Sp & Sn & Sp & Sn & Sp & Sn & Sp & Sn & Sn \\
\hline Lab1 & $\mathbf{9 7 . 5}$ & $\mathbf{0 . 9 6}$ & $\mathbf{9 8 . 3}$ & $\mathbf{0 . 9 8}$ & $\mathbf{8 9 . 7}$ & $\mathbf{0 . 8 8}$ & $\mathbf{5 8 . 2}$ & $\mathbf{0 . 5 5}$ & $\mathbf{7 7 . 6}$ & $\mathbf{0 . 7 5}$ \\
Lab2 & 94.5 & 0.92 & 95 & 0.93 & 84.6 & 0.84 & 54.3 & 0.52 & 74.7 & 0.73 \\
Lab3 & 93.3 & 0.92 & 93.8 & 0.90 & 82 & 0.82 & 53.6 & 0.52 & 73.4 & 0.71 \\
HSV1 & $\mathbf{9 5 . 6}$ & $\mathbf{0 . 9 4}$ & $\mathbf{9 6 . 7}$ & $\mathbf{0 . 9 4}$ & $\mathbf{8 6}$ & $\mathbf{0 . 8 6}$ & $\mathbf{5 5 . 5}$ & $\mathbf{0 . 5 4}$ & $\mathbf{7 6 . 9}$ & $\mathbf{0 . 7 5}$ \\
HSV2 & 93.1 & 0.93 & 93.4 & 0.93 & 83.1 & 0.83 & 53.6 & 0.53 & 73.9 & 0.73 \\
HSV3 & 90.4 & 0.88 & 90.8 & 0.88 & 80.5 & 0.82 & 51.3 & 0.49 & 70.4 & 0.68 \\
\hline
\end{tabular}

Table 5: Average percentage classification ( $\mathrm{Sn}$ ) using the best multi-models in Lab and HSV in comparison with existing approaches.

\begin{tabular}{cccccc} 
& DB1 & DB2 & DB3 & DB4 & DB5 \\
\hline Lab1 (proposed method) & 97.5 & 98.3 & 89.7 & 58.2 & 77.6 \\
(Qazi et al. 2011), in Lab & 97.2 & 96.5 & 88 & 52.1 & 78.5 \\
HSV1 (proposed method) & 95.6 & 97.8 & 86 & 55.5 & 76.9 \\
(Qazi et al. 2010), in IHLS & 95.4 & 97.4 & 84.1 & 49.3 & 77.1 \\
(Verdoolaege et al. 2012), in RGB & 92.2 & 94.7 & 78.5 & 59.6 & 75.1 \\
(Kwitt et al. 2011), in RGB & 91.5 & 94.1 & 79.8 & 55.4 & 74.9 \\
\hline
\end{tabular}

Table 6: Average percentage classification Sn and runtime (in minutes) on DB2, using Rao distance, ML based divergence and Monte-carlo based KL divergence as similarity measure for Lab1 and HSV1 multi-models.

\begin{tabular}{ccccccc}
\hline & \multicolumn{2}{c}{ Rao } & \multicolumn{2}{c}{$\mathrm{KL}_{\mathrm{ML}}$} & \multicolumn{2}{c}{$\mathrm{KL}_{\mathrm{MC}}$} \\
\hline & Sn & Runtime & Sn & Runtime & Sn & Runtime \\
\hline Lab1 & 98.3 & $4.9 \mathrm{~min}$ & 98 & $40.5 \mathrm{~min}$ & 97.2 & $60 \mathrm{~min}$ \\
\hline HSV1 & 96.7 & $6.5 \mathrm{~min}$ & 95.9 & $44.3 \mathrm{~min}$ & 96.2 & $63 \mathrm{~min}$ \\
\hline
\end{tabular}

Table 7: Classifier performances for full feature vector (spatial structure for luminance and chrominance)

\begin{tabular}{ccccccc}
\hline & \multicolumn{2}{c}{ DB1 } & \multicolumn{2}{c}{ DB2 } & \multicolumn{2}{c}{ DB3 } \\
\hline & Sn & Sp & Sn & Sp & Sn & Sp \\
\hline Lab1 & 86.9 & 0.83 & 90 & 0.88 & 75.8 & 0.78 \\
\hline HSV1 & 84.5 & 0.82 & 89.6 & 0.88 & 72.2 & 0.70 \\
\hline
\end{tabular}


to construct a circular/linear bivariate model for chrominance such as $\{$ vonMises, Weibull $\}$ or $\{$ vonMises, Gamma (see Table 3).

Regarding the first part of our experiments, Table 4 presents color texture classification performance in Lab and HSV color spaces respectively, using different multimodels on DB1, DB2, DB3, DB4 and DB5. We note that:

- Lab1: refers to a multi-model that considers CopMGam (Table 3) for the luminance model $M_{L}$ and CopMWbl for the chrominance model $M_{C_{r}}$ in Lab color space.

- Lab2: refers to CopMWbl for $M_{L}$ and CopMGam for $M_{C_{r}}$ in Lab color space.

- Lab3: refers to CopMGam model for $M_{L}$ and the bivariate model $\{$ Gamma,Weibull $\}$ for $M_{C_{r}}$ in Lab color space.

- HSV1: refers to CopMGam for $M_{L}$ and the bivariate model $\{$ vonMises, Weibull $\}$ for $M_{C_{r}}$ in HSV color space. (vonMises for the Hue marginal, and Weibull for the Saturation marginal)

- HSV2: refers to CopMWbl for $M_{L}$ and the bivariate model $\left\{\right.$ vonMises, Gamma\} for $M_{C_{r}}$ in HSV color space.

- HSV3: refers to CopMGam for $M_{L}$ and and the bivariate model \{Weibull,Gamma\} for $M_{C_{r}}$ in HSV color space.

We remark that in Lab, and for all the five databases, best performance is achieved using the multi-model Lab1. This indicates that the spatial structure along with the inter-band dependency are better characterized using the Gaussian copula in conjunction with Gamma marginals (CopMGam). This also indicates that the dependency between "a" and "b" chrominance components is better characterized using the Gaussian copula in conjunction with Weibull marginals (bivariate CopMWbl). For HSV, the multi-model HSV1 leads to the best performance in all databases. This is due to the ability of CopMGam to model spatial structure and inter-band dependence for luminance, and the circular/linear bivariate \{vonMises, Weibull $\}$ to characterize the chrominance dependency.

We next would like to compare between the perceptually uniform and perceptual subfamilies of LC color spaces, via their respective representatives Lab and HSV spaces. Considering the best multi-models in each case (Lab1 for Lab and HSV1 for HSV), we clearly observe from Table 4 that Lab representation outperforms the HSV one. This is a fair comparison even the models $M_{L}$ and $M_{C_{r}}$ are not the same in multi-models Lab1 and HSV1, since these latters are the best relatively the nature of the color space. Then, if Lab1 outperforms HSV1, we can conclude that in best modeling case the Lab representation is better than the HSV one.
These results are explained by the better luminancechrominance representation of Lab. In other words, we can say that Lab offers more decorrelation property between luminance and chrominance, which is the central interest of our approach. We do not ignore that percentage classification and precision rates are lower for large databases DB3, DB4 and DB5.

Regarding the effectiveness of our approach in comparison with existing ones, i.e uni-modeling in LC and RGB color spaces, Table 5 shows average percentage classification $\mathrm{Sn}$ of textures of DB1, DB2, DB3, DB4 and DB5 using our best multi-models (Lab1 and HSV1) against the four following approaches:

- (Qazi et al. 2010), in Lab: Linear prediction models based characterization in the Lab color space,

- (Qazi et al. 2011), in IHLS: Linear prediction models based characterization in the improved hue luminance saturation (IHLS ) color space,

- (Verdoolaege et al. 2012), in RGB: Dependence between R, G and B color components using the MGGD model,

- (Kwitt et al. 2011), in RGB: Dependence between R, $\mathrm{G}$ and $\mathrm{B}$ using the t-copula based multivariate Weibull model,

We observe from Table 5 that our Lab1 proposed model leads to better performance when compared with the linear prediction modeling (Qazi et al. 2010) and (Qazi et al. 2011). Results presented in the latter studies are the best of our knowledge, but it should be highlighted that the characterization of both luminance and chrominance channels by using the $2-\mathrm{D}$ auto regressive (AR) models with the same order, seems a limitation because the two channels are independent. However, it is noteworthy to precise that the results shown in Table 5 for the two studies consider only the case when authors use the spatial structure and chrominance information without adding the color information (3-D color histograms) in the characterization. We note that there is a slight performance for this later for DB5 database and for (Verdoolaege et al. 2012) for DB4 database. We also remark from the same table the superiority of the characterization in LC color spaces compared to the RGB one except for DB4. This is theoretically confirmed since considering the image as two separable luminance and chrominance information is more intuitive and correlated with the human perception than the RGB space which considers the color as an addition of the primary colors red, green and blue. Here, we also note that (Verdoolaege et al. 2012) considered uniquely the dependency between R, G and B components (inter-channel only), and that (Kwitt et al. 2011) considered the R, G and $\mathrm{B}$ dependency besides the inter-band one (for the three components). As we stressed in the introduction, extending these models to take into account jointly intra-band, 
inter-band and inter-channel dependencies leads to highdimensionality, due to the huge dependence between $\mathrm{R}, \mathrm{G}$ and $\mathrm{B}$ components, and to the fact that RGB representation offers no separation between spatial structure and chrominance.

Another contribution of our approach is the definition of a closed form expression for the Rao distance in the variety of copula based probability densities (El Maliani et al. 2011). As it is known, deriving a similarity measure in the case of copula based multivariate models is a challenging task. For this, in (Kwitt et al. 2009) and (Sakji-Nsibi et al. 2010), authors proposed a Monte-carlo approximation of the KL divergence. But, this approach is computationally expensive and is not deterministic, since the KL divergence differs depending on the random number generation. An alternative approach was proposed in (Kwitt et al. 2011), using the maximum likelihood (ML) selection rule as a similarity measure. This significantly reduces the execution time in comparison with the Monte-carlo based similarity measurement. A more attractive expression of the KL divergence is provided in (Lasmar et al. 2012), where the separability between the copula space and the marginal space is used to derive a closed-form and fast KL based similarity measurement. However, KL divergence does not satisfy properties of symmetry and triangular inequality, and thus is not a distance in the right sense of the parametric manifold. Table 6 shows average percentage classification along with the execution time using both similarity measures (Rao and KL) for the textures of DB2. We recall that according to equation 37, we need expression of the Rao distance for marginals and the used copula. Expressions of the Rao distance for Weibull, Gamma and vonMises can be found respectively in (El Maliani et al. 2011), (Reverter et al. 2003) and (Ceolini et al. 2010). We can see that the proposed similarity measure slightly outperforms the ML based and Monte-Carlo based approach in term of percentage classification. However, the improvement is more significant in term of runtime which is 9 times and 12 times less than the ML based and Monte-carlo based approaches respectively. We note that the experiments were done using Matlab environment on an HP Compaq equipped with an Intel Core 2 Duo CPU at $3 \mathrm{GHZ}$ workstation

Finally, we want to validate experimentally the hypothesis made in introduction about dimensionality reduction when we consider multi-modeling in LC color spaces. We compare our approach to the full dependence based approach of subsection 2.1. Table 7 shows performance of the classifier for multi-models Lab1 and HSV1 with full feature vectors length. It can be seen from these results that the high parametrisation does not improve performances, but on contrary it makes them decrease by about $10 \%$. Separability between luminance and chrominance helps then to avoid redundancy in characterization which is the case in RGB where spatial structure resides in the three color components.

\section{Conclusion}

We proposed in this paper a multi-model based approach for color textures characterization in the LC color spaces. This approach takes into account the separability between luminance and chrominance. We especially took care of the circular/linear nature of chrominance components. We also addressed the dimensionality reduction issue for the study of the full dependence in term of intra-band, inter-band and inter-channel relation between coefficients color component subbands. Results on five wide range of databases show the superiority of the proposed approach in comparison with the existing unimodeling approaches. We know that our method as any compared methods, presents some limitations when using large databases and also against rotation and illumination effects. Furthermore, considering the runtime issue, results show the effectiveness of the Rao geodesic distance in comparison with the Kullback-leibler divergence.

\section{References}

[Verdoolaege et al. 2012] G. Verdoolaege and P.Scheunders, "On the Geometry of Multivariate Generalized Gaussian Models," Journal of Mathematical Imaging and Vision, vol. 43, pp. 180-193, 2012 .

[Kwitt et al. 2011] R. Kwitt, P. Meerwald and A. Uhl, "Efficient Texture Image Retrieval Using Copulas in a Bayesian Framework," IEEE transactions on image processing, vol. 20, pp. 2063-2077, 2011.

[Seetharama et al. 2014] K. Seetharaman, M. Jeyakarthic, "Statistical distributional approach for scale and rotation invariant color image retrieval using multivariate parametric tests and orthogonality condition," Journal of Visual Communication and Image Representation, vol. 25, pp. 727-739, 2014.

[Maliani et al. 2012] A.D. El Maliani, M. El Hassouni, Y. Berthoumieu, D. Aboutajdine, "Multi-model Approach for Multicomponent Texture Classification," Image and Signal Processing Lecture Notes in Computer Science, vol. 7340, pp. 36$44,2011$.

[Qazi et al. 2011] I. Qazi, O. Alata, J.C. Burie, A. Moussa and C.F. Maloigne, "Choice of a pertinent color space for color texture characterization using parametric spectral analysis," Pattern Recognition, vol. 44, pp. 16-33, 2011.

[Qazi et al. 2010] I. Qazi, O. Alata, J.C. Burie and C.F. Maloigne "Color spectral analysis for spatial structure characterization of textures in IHLS color space," Pattern Recognition, vol. 43, pp. $663-675,2010$.

[Rakvongthai et al. 2013] Y. Rakvongthai, S. Oraintara "Statistical texture retrieval in noise using complex wavelets," Signal Processing: Image Communication, vol. 28, pp. 14941505, 2013.

[DeYoe et al. 1996] E. DeYoe and D. van Essen, "Concurrent processing streams in monkey visual cortex". Trends. Neurosci., 11:219226, 1996.

[Mojsilovic et al. 2000] A. Mojsilovic, J. Kovacevic, D. Kall, R. Safranek, and S. Ganapathy, "Matching and retrieval based on the vocabulary and grammar of color patterns". IEEE Transactions on Image Processing, 9(1):3854, 2000.

[Sarifuddin et al. 2005] M. Sarifuddin and R. Missaoui, "A New Perceptually Uniform Color Space with Associated Color Similarity Measure for Content Based Image and Video Retrieval", Proceedings of Multimedia Information Retrieval Workshop, 28th annual ACM SIGIR Conference, pp. 1-8, 2005.

[Simoncelli 1997] E. P. Simoncelli, "Statistical models for images: Compression, restoration and synthesis", IEEE Conference on Signals, Systems \& Computers, 1997., Conference Record of the Thirty-First Asilomar, pp. 673-678, 1997. 
[Kwitt et al. 2009] R. Kwitt and A. Uhl, "A joint model of complex wavelet coefficients for texture retrieval," in Image Processing, 2009. ICIP 2009. 16th IEEE International Conference, 2009, pp. $1877-1880$.

[Boubchir et al. 2010] L. Boubchir, A. Nait-Ali and E. Petit, "Multivariate statistical modeling of images in sparse multiscale transforms domain," in Proceeding of the 17th IEEE International Conference on Image Processing (ICIP'10), 2010, pp. 18771880.

[Lasmar et al. 2010] N. Lasmar and Y.Berthoumieu, "Multivariate statistical modeling for texture analysis using wavelet transforms," in Proceeding of the IEEE International Conference on Acoustics Speech and Signal Processing (ICASSP'10), 2010 pp. $790-793$.

[Sakji-Nsibi et al. 2010] S. Sakji-Nsibi and A. Benazza-Benyahia, "Fast scalable retrieval of multispectral images with kullbackleibler divergence,textquotedblright in Image Processing, 2010. ICIP 2010. 17th IEEE International Conference, 2010, pp. 2333-2336.

[Nelsen 2006] R. B. Nelsen, An Introduction to Copulas. Springer Series in Statistics. Springer, second edition, 2006.

[Sklar et al. 1959] M. Sklar, "Fonctions de répartition à $n$ dimensions et leurs marges,". Publications de l'institut de Statistique de l'Université de Paris. vol. 8, pp. 229-231, 1959.

[Joe. 1997] H. Joe, Multivariate Models and Dependence Concepts. Monographs on Statistics and Applied Probability. Chapman \& Hall, 1997.

[Durrleman et al. 1959] V. Durrleman, A.Nikeghbali, and T. Roncalli, "Which copula is the right one," Groupe de Recherche Operationnelle, Credit Lyonnais, 2000.

[Lasmar et al. 2012] N. Lasmar, Y. Berthoumieu, "Gaussian Copula Multivariate Modeling for Image Texture Retrieval Using Wavelet Transforms," submitted to IEEE transactions on image processing.

[Bishop 2006] C. M. Bishop, "Pattern recognition and machine learning", Springer, 2006

[Mallat 1999] S. G. Mallat, "A wavelt tour of signal processing", 2nd edition, San Diego: Academic press, 1999.

[El Maliani et al. 2011] A. D. EL Maliani, M. EL Hassouni, N. Lasmar and Y. Berthoumieu, "Color texture classification using rao distance between multivariate copula based models," in Proceedings of the 14th international conference on Computer Analysis of Images and Patterns, CAIP11, pp. 498505, 2011.

[Reverter et al. 2003] F. Reverter and J.M. Oller, "Computing the Rao distance for Gamma distributions," Journal of Computational and Applied Mathematics, vol. 157, pp. 155-167, 2003.

[Ceolini et al. 2010] S. Ceolini and E. R. Hancock, "Characterising facial gender difference using fisher-rao metric," in Proceedings of the 2010 20th International Conference on Pattern Recognition, ICPR10, pp. 43084311, 2010.

[Do et al. 2002] M. Do and M. Vetterli, "Wavelet-based texture retrieval using generalized Gaussian density and Kullback-Leibler distance," IEEE transactions on image processing, vol. 11, pp. 146-158, 2002.

[Rangaswamy et al. 2002] M. Rangaswamy, and D. Weiner, Donald and A. Ozturk, "Non-Gaussian random vector identification using spherically invariant random processes," IEEE Transactions on Aerospace and Electronic Systems, vol. 29, pp. 111-124, 1993.

[Vistex] "MIT vision and modeling group," [Online], Available from: http://vismod.media.mit.edu.

[Outex] "Available from: http://www.outex.oulu.fi/,".

[ALOT] Amsterdam Library of Textures. [Online]. Available from: http://staff.science.uva.nl/ aloi/public_alot

[STex] Salzburg Textures [Online]. Available from: http://wavelab.at/sources/STex

[Ojala et al.] T. Ojala, M. Pietikinen, T. Menp, J. Viertola, J. Kyllnen, and S. Huovinen. "Outex - new framework for empirical evaluation of texture analysis algorithms". In 16th International Conference on Pattern Recognition, Quebec, Canada, August 2002 .
[Permuter et al. 2006] H. Permuter, J. Francos, and I. Jermyn, "A study of gaussian mixture models of color and texture features for image classification and segmentation," Pattern Recognition, vol. 39(4), pp. 695-706, 2006.

[Atkinson et al. 1981] C. Atkinson, A.F. Mitchell, "Rao's distance measure". Sankhy: The Indian Journal of Statistics, Series A, 345-365. 1981

[Kingsbury 1998] N. Kingsbury, "The Dual-Tree Complex Wavelet Transform: A new Technique for Shift-Invariance and Directional Filters," in Proceedings of the 8th IEEE DSP Workshop, Aug. 1998, pp. 9-12. 